International Journal of Agriculture, Environment and Bioresearch

Vol. 5, No. 05; 2020

ISSN: $2456-8643$

\title{
IMPACT OF COMPLEX CARBOHYDRATES ON HUMAN HEALTH
}

\author{
Kumari Sushma Saroj \\ Department of Zoology ,Dr. L.K.V.D. College, Tajpur, Samastipur ,L.N. Mithila University, Darbhanga, India \\ https://doi.org/10.35410/IJAEB.2020.5552
}

\begin{abstract}
Carbohydrates are an important part of a nutritional diet. The healthiest sources include complex carbohydrates because of their blunted effects on blood glucose. Whole grain consumption is associated with diminished risk of serious, diet-related diseases, which are major problems in wealthy industrialized economies and are emerging in developing countries demographic and nutrition transition. Carbohydrates are important contributors to the health benefits of whole grains. Insoluble non-starch polysaccharides (NSP) are effective laxatives. Soluble NSP lower plasma cholesterol and so can reduce heart disease risk but the effect is inconsistent. Processing seems to be an important contributor to this variability and other grain components may be involved. However, starch not digested in the small intestine (resistant starch, RS) appears to be as important as NSP to large bowel function. Native Africans at low risk of diet-related disease through consumption of unrefined cereals may actually have relatively low fibre intakes. While NSP are effective faecal bulking agents, they are fermented to a very variable extent by the large bowel microflora. In contrast, RS seems to act largely through the short chain fatty acids (SCFA) produced by these bacteria. One SCFA (butyrate) appears to be particularly effective in promoting large bowel function and RS fermentation appears to favour butyrate production. Animal studies show that dietary RS lowers diet-induced colonocyte genetic damage and chemically-induced large bowel cancer which correlates with increased butyrate. These effects in long term could contribute to a lower risk of cancer and ulcerative colitis. Cereal grain oligosaccharide (OS) may also function as prebiotics and increase the levels of beneficial bacteria in the large bowel.
\end{abstract}

Keywords: Starch, Oligosaccharides, Non-Starch Polysaccharides, Cereals, Human Health.

\section{INTRODUCTION}

Cereal based foods have been staples for human's race since agriculture evolved. Cereal grains contain the macronutrients (protein, fat and carbohydrate) required by humans for growth, development and maintenance. They also supply important minerals, vitamins and other phytonutrients essential for optimal health. However, it is becoming apparent that cereals in general have the potential for health enhancement beyond the simple provision of these nutrients and phytonutrients as their consumption can lower the risk of significant diet-related diseases. Morbidity and mortality from non-infectious diseases are significant problems in not only in affluent, but also in developed economies and include cardiovascular disease (CVD), Type 2 diabetes and certain type of cancers [1]. Most of the pericarp-seed coat, aleurone and germ 
containing (fibre, micronutrients, fat and protein) are retained in the whole grain while they have been removed by milling, polishing and processing in refined foods, leaving the starchy endosperm. Studies have shown that consumption of whole grain products is associated with lowered risk of CVD and diabetes [2]. Greater intake of whole grain foods is associated also with lesser obesity $[3,4]$. Two hypotheses have been proposed to account for this conundrum. The first is that the whole grains contain a complete portfolio of bioactive components which provide comprehensive protection for the major disease which have been identified in population studies

\section{Types of Carbohydrates}

a. Simple carbohydrates

b. Complex carbohydrates

\section{Carbohydrates can also be Classified on the Basis of Their Chemical Composition}
a. Monosaccarides
b. Disaccharides
c. Oligosaccharides
d. Polysaccharides

\section{GRAIN CARBOHYDRATES AND HUMAN HEALTH}

Dietary carbohydrates can be divided very simply into two major groups solely on the basis of their susceptibility to digestion by human small intestinal enzymes. Humans possess a suite of small intestinal digestive enzymes but only amylase that can attack a complex carbohydrate. This enzyme can hydrolyze only one significant dietarypolysaccharide starch. This specificity depends on the presence of 1,4 glucosidic links in the target polysaccharide. All of the other major complex carbohydrates likeoligosaccharides (OS) and non-starch polysaccharides (NSP)) seem to resist human small intestinal enzymic digestion completely. NSP are the principal components of dietary fibre and the lack of small intestinal digestibility explains their principal physiological properties. It must be emphasized that this solubility may be demonstrated under conditionswhich do not occur in the human small intestine [5]. Nevertheless, the terms "soluble fibre" and "insoluble fibre"' have entered into common usage and also serve to segregate NSP on one of their best documented physiological effects of reducing plasma cholesterol, an established risk factor for CVD [6]. On the other carbohydrates found in physiologically significant quantities in some grains, OS are a relatively known quantity in terms of the health 
Vol. 5, No. 05; 2020

ISSN: $2456-8643$

benefits of cereals. They contribute to the dietary fibre content of grains and have health benefits.

Starch is the most important polysaccharide in nearly all seeds grown and used as human foods. Slow digestion of starch leads to a low glycemic response (GR) while reducing total small intestinal amylolysis increases RS. Amylose is slower to gelatinize and quicker to retrograde than amylopectin and so processed food products have higher RS.

\section{Dietary Fibre and Health Outcomes}

It is essential that any potential health benefits of foods or food components be substantiated to ensure their effectiveness and sustain consumer confidence. For dietary fibre there are two specific instances where the positive outcomes of greater consumption have been established namely laxation and plasma cholesterol reduction.

\section{NSP and Laxation}

Burkitt and Co-workers [7] in Africa noted that black Africans were at much lower risk of what have become known as the "diseases of affluence" than Europeans living in the same environment. The difference was ascribed to the greater consumption of unrefined foods by the natives. With time, research emphasis shifted to the apparently greater fibre content of these foods and the development of the so-called 'roughage" model of dietary fibre. This model hinges on the resistance of NSP to human small intestinal digestive enzymes which helps to explain the well-recognizeddose dependent effects of fibre rich foods in increasing faecal bulk. This bulking is taken as the mechanism of action of what is probably the best documented effect of dietary fibre promotion of regularity.

\section{Soluble NSP and Plasma Cholesterol}

CHD is a disease characterized by infiltration of cholesterol into the major arteries of the heart. Atherosclerosis leads to a progressive occlusion of the circulation resulting in impaired cardiac function and, if unchecked, tissue necrosis and death. CHD has a number of risk factors which cannot be altered. Modifiable risk factors include smoking, physical activity and raised plasma low-density lipoprotein (LDL) cholesterol. LDL is the major plasma vehicle for transporting cholesterol to the tissues.

The effectiveness of this approach has to be quantified relative to drugs. The potential of grain components to assist in risk reduction isfairly self-evident, given the relationship between whole grain consumption and lowered CHD risk. It was thought originally that dietary insoluble fibre was responsible, which reduced plasma cholesterol. The proposition was based on the observations that plant fibre concentrates and isolates bind bile acids in vitro.

\section{Starch and Human Health}

The paradox between food consumption and relative risk can be reconciled by the facts that the African ate considerably more unrefined starch than the Europeans and that their cooking practices were rather different. In particular, the Africans ate maize porridge, which had been 
cooked in water and then cooled and stored for some time. While cooking increases the digestibility of starch and other nutrients, the cooling and storage leads to retrogradation of starch and the formation of RS. Human studies have confirmed that the stored cooked maize gruel was high in RS relative to the fresh product. The importance of controlling the rate of small intestinal starch digestion to lower the GR is accepted. For optimal large bowel health, combinations of RS and NSP may be optimal.

RS as a health-related food component is probably quite simple. Starch has the potential to be hydrolyzed completely to glucose in the small intestine. In omnivores, this fermentation occurs principally in the large bowel. These data point to an important difference between RS and NSP. The human caecum and colon are home to a numerically large, taxonomically diverse and metabolically active, bacterial population. Most of the bacteria in adult humans are nonpathogenic and ferment unabsorbeddietary carbohydrates. These organisms resemble those found in the large bowel of obligate herbivores in metabolic capacity and end products, i.e. gases $\left(\mathrm{H}_{2}\right.$, $\mathrm{CO}_{2}$ and $\mathrm{CH}_{4}$ ) and short chain fatty acids (SCFA).

\section{Short Chain Fatty Acids and Health Effects of Complex Carbohydrates}

Health benefits of complex carbohydrates accrue from the short chain fatty acids (SCFA) produced in the large bowel [8]. SCFA have a number of general effects including the direct acidification of digesta. This lowering of $\mathrm{pH}$ leads to the ionisation of potentially cytotoxic compounds including biogenic amines and ammonia [9]. SCFA are absorbed on passage of the faecal stream and less than $10 \%$ of those produced appear in excreted faeces. The absorbed SCFA are metabolised by the viscera and contribute to their energy needs. Butyrate is the preferred metabolic substrate for colonocytes. Its metabolism is thought to drive the uptake of cations and water leading to water salvage. Incorporation of RS into the oral rehydration solution accelerates recovery and lowers net water loss in patients with watery diarrhoea . Butyrate is also believed to promote a normal phenotype in colonocytes through the repair of damaged DNA and induction of programmed cell death in transformed cells. These actions are thought to lower the risk of colorectal cancer and have been shown in cultured transformed cells. SCFA have a number of other positive effects on large bowel health with butyrate being the most potent.

\section{CONCLUSION}

There is strong evidence that cereal polysaccharides have benefits in the control and prevention of human diseases. Their capacity to improve health status is established reasonably well in some areas, for example, soluble NSP and plasma cholesterol control.Understanding the relationships between the metagenome, carbohydrates, SCFA and health will offer great opportunities to improve health. This should include obtaining an understanding of the interactions between cereal OS and the microflora. The new cereal cultivars are aimed at improving public health in important areas, including diabetes. These new technologies will also help to unravel the relationships between gut microflora, food polysaccharides and health. 
Vol. 5, No. 05; 2020

ISSN: $2456-8643$

\section{REFERENCE}

Jacobs Jr, DR and Gallaher DD Whole grain intake and cardiovascular disease A Review. CurrAther Rep6 414-423 (2004)

Fenech M, Noakes M, Clifton P and Topping D Aleurone flour increases red cell folate and lowers plasma homocyst(e)in substantially in humans. British Jour Nutr93 353-360 (2005)

Jemal A, Ward E, Hao Y and Thun M Trends in the leading causes of death in the United States, 1970-2002. JourAmer Med Assoc2941255-1259 (2005)

Le Leu RK, Brown IL, Hu Y, Bird AR, Jackson M, Esterman A and Young GP A symbiotic combination of resistant starch and Bifidobacteriumlactis facilitates apoptotic deletion of carcinogen damaged cells in rat colon. Journal of Nutrition 135996-1001 (2005)

Topping DL Soluble fiber polysaccharides: effects on plasma cholesterol and large bowel shortchain fatty acids. Nutr Rev 49195-203 (1991)

Clearfield M Statins and the primary prevention of cardiovascular events. CurrAtherosc Rep8 390- 396 (2006)

Burkitt DP Some diseases characteristic of western civilisation. British Medical Journal2 274276 (1973)

Topping DL and Clifton PM Short-chain fatty acids and human colonic function: roles of resistant starch and nonstarch polysaccharides. Physiol Rev81 1031- 1064 (2001)

Visek WJ Diet and cell growth modulation by ammonia. Amer Jour ClinNutr31 (Suppl. 10) S216-S220 (1978) 